\title{
Deltonin Ameliorates Cerebral Ischemia/Reperfusion Injury in Correlation with Modulation of Autophagy and Inflammation
}

This article was published in the following Dove Press journal:

Neuropsychiatric Disease and Treatment

\author{
Yi Zhang' \\ Zhiming Tian' \\ Hongyan Wan' \\ Wen Liu' \\ Fanping Kong ${ }^{2}$ \\ Guoping $\mathrm{Ma}^{3}$ \\ 'Cerebral Vascular Center, Zhongda \\ Hospital, Southeast University, Nanjing \\ City, Jiangsu Province 210044, People's \\ Republic of China; ${ }^{2}$ Department of \\ Neurology, Fu-Ning People's Hospital, \\ Yancheng City, Jiangsu Province 224400, \\ People's Republic of China; ${ }^{3}$ Department \\ of Neurology, Tian-Shui First People's \\ Hospital, Tianshui City, Gansu Province \\ 741000, People's Republic of China
}

Correspondence: Fanping Kong Department of Neurology, Fu-Ning People's Hospital, No. III Bucheng Street, Yancheng City, Jiangsu Province 224400, People's Republic of China Email kongfanping_07@I63.com

\section{Guoping Ma}

Department of Neurology, Tian-Shui First People's Hospital, No. 105 Jianshe Road,

Tianshui City, Gansu Province 741000,

People's Republic of China

Email ahygmcynbiis9@163.com
Introduction: Deltonin, an active component extracted from Dioscorea zingiberensis C.H. WRIGHT, was widely utilized in traditional Chinese medicines. It has been shown to have anticancer functions such as colon cancer, breast cancer, and head and neck squamous carcinoma. Herein, we will investigate the role of deltonin in cerebral ischemia/reperfusion injuries.

Methods: Ly294002 and anisomycin were used as inhibitors to monitor the effects of deltonin. Middle cerebral artery occlusion I/R model was constructed. Infarct volumes, neurological deficits and brain water contents were evaluated under different conditions Rotarod test, ELISA, and Western blotting were carried to investigate the effects in vitro.

Results: We found that deltonin in ischemia/reperfusion $(\mathrm{I} / \mathrm{R})$ rats greatly enhanced brain damages as well as neurological functions through up-regulating p-Akt and p-mTOR as well as inhibiting the expressions of LC3-II/LC3-I, Beclin-1, IL-1, TLR4, and p-p38. Deltonin exerted neuroprotection effect through relieving autophagy activity by regulating PI3K/Akt/ mTOR signaling. Deltonin suppressed inflammation reactions through modulation TLR4/ p38/MAPK signaling as well.

Conclusion: Overall, our data suggested that deltonin could suppress ischemic brain injury by regulating autophagy and inflammation during $\mathrm{I} / \mathrm{R}$. Deltonin can be a potential therapeutic method for patient with $\mathrm{I} / \mathrm{R}$.

Keywords: deltonin, cerebral I/R, MCAO/R, autophagy, inflammation

\section{Introductions}

Ischemic stroke is resulted from the occlusion of major cerebral arteries. It is a devastating disease, with a major cause of death and disability worldwide. Deltonin is a kind of steroidal saponin, with a chemical structure of $\mathrm{C}_{45} \mathrm{H}_{72} \mathrm{O}_{17}$. Deltonin, an active component extracted from Dioscorea zingiberensis C.H. WRIGHT, was widely utilized in traditional Chinese medicines. It has been shown to have anticancer functions such as colon cancer, ${ }^{1}$ breast cancer, ${ }^{2}$ and head and neck squamous carcinoma. ${ }^{3}$ Xie et al demonstrated that deltonin was associated with the cell apoptosis and autophagy in head and neck squamous carcinoma FaDu cell. ${ }^{3}$ Deltonin may have a strong connection with cell apoptosis and autophagy. Herein, we will investigate the role of deltonin in cerebral ischemia/reperfusion injuries.

Autophagy is a cellular function that is related to cell homeostasis, defenses and adaptations to different environments. Regarding ischemia, many previous reports have revealed that autophagy participates actively in the developments of cerebral ischemia, ${ }^{4}$ including global ischemia, ${ }^{5}$ focal ischemia, ${ }^{6}$ and also 
hypoxia-ischemia. ${ }^{5,7}$ Neuroinflammation caused by ischemia/reperfusion injuries brings damages to the neuron cells. Therefore, the inhibitions of autophagy and inflammation are critical to prevent the neuro-degenerative damages after focal cerebra $\mathrm{I} / \mathrm{R}^{8}$

$\mathrm{PI} 3 \mathrm{~K} / \mathrm{Akt} / \mathrm{mTOR}$ pathway has been proved to play central roles in ischemia-reperfusion $(\mathrm{I} / \mathrm{R})$ injuries. For instance, Zhang reported that sevoflurane post-conditioning protected rat hearts against ischemia-reperfusion injury through the activation of PI3K/AKT/mTOR signaling. ${ }^{9}$ Chen also reported the neuroprotective effects of brainderived neurotrophic factor mediated by autophagy through the PI3K/Akt/mTOR pathway. ${ }^{10}$ In addition, TLR4/p38/MAPK pathway was also reported to be involved in the neuro inflammations in microglia. In 2016, Swaroop reported that HSP60 could regulate IL-1 $\beta$-related microglial inflammatory reactions through TLR4-p38/MAPK. In this paper, we aim to reveal the functional mechanisms of deltonin, $\mathrm{PI} 3 \mathrm{~K} / \mathrm{Akt} / \mathrm{mTOR}$ pathway TLR4-p38 MAPK pathway in the autophagy and inflammation caused by cerebral ischemia/reperfusion injury, as well as their inner associations.

\section{Methods and Materials}

\section{Reagents}

Deltonin with a purity of over $98 \%$ was generated as previously described and its purity determined by high-performance liquid chromatography ( $>98 \%$ ) according to Tong et al. ${ }^{1}$ Figure $1 \mathrm{~A}$ shows the chemical structure of deltonin.
LY294002, anisomycin and TTC reagents were obtained from Sigma, USA. Assays to detect IL-6, IL-10, TNF- $\alpha$, and iNOS were provided by Jiancheng Bio. Ins., China. Rabbit anti-Akt, anti-Phospho-Akt (p-Akt), anti-mTOR, anti-Phospho-mTOR (anti-p-mTOR), anti-P38, antiPhospho-p38 (anti-p-P38), anti-LC3, anti-Beclin-1, antimicrotubule-associated protein (anti-MAP-2), anti-TLR4 and anti-IL-1 were obtained from Abcam, USA). Rabbit anti-GAPDH was purchased from Beijing Zhongshan Jinqiao Bio., China. Horseradish peroxidase linked goat anti-rabbit IgG was provided by Santa Cruz Biotechnology, US.

\section{Animals}

Male Sprague-Dawley rats (200-220g) were bought from the Experimental Animal Center of Fu-Ning People's Hospital, Yancheng, China. Rats were maintained at $22-24^{\circ} \mathrm{C}$ with a $12-\mathrm{hr}$ light and $12-\mathrm{h}$ dark cycle. They were free to eat and drink. Animal Care and Use Committee of Fu-Ning People's Hospital approved our researches, which were conducted by strictly flowing the NIH Guidelines for the Care and Use of Laboratory Animals.

\section{Focal Cerebral I/R Model}

MCAO/R (middle cerebral artery occlusion I/R), 2/24h ${ }^{11}$ was conducted firstly by anesthetizing the rats with $10 \%$ $(\mathrm{w} / \mathrm{v})$ chloral hydrate $(350 \mathrm{mg} / \mathrm{kg})$ intraperitoneally at $37^{\circ} \mathrm{C}$. After incising the skins and muscles, left common

A

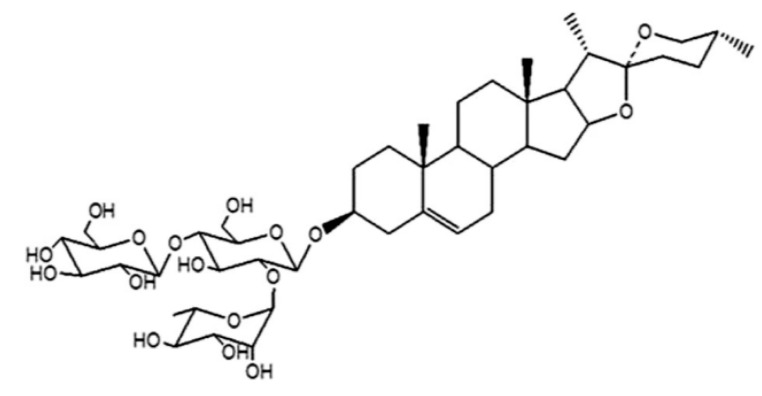

B

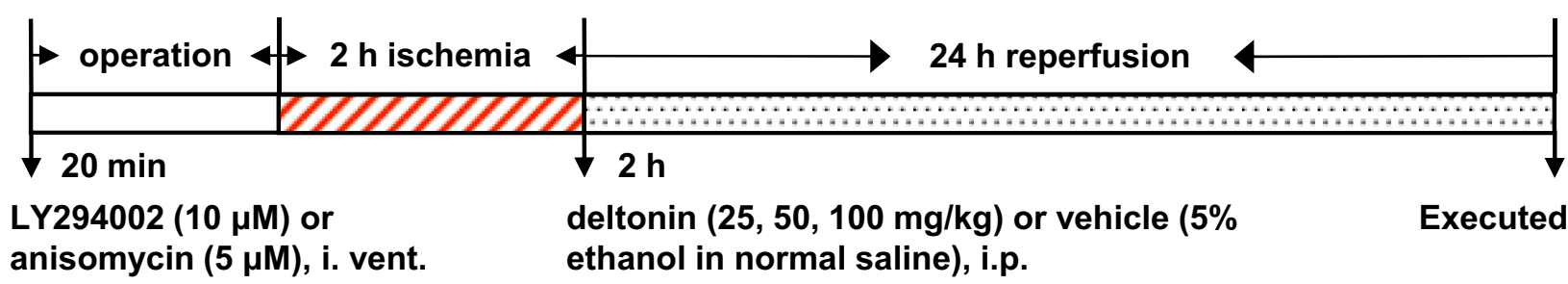

Figure I Deltonin's chemical structure (A) and experiment protocol (B). 
carotid artery (CCA) was clipped, and external carotid artery (ECA) was ligatured. A monofilament was inserted from CCA to internal carotid artery (ICA). After $2 \mathrm{hrs'}$ MCAO, reperfusion was conducted via removing the monofilament. The sham group had I/R surgery, without the insertion of nanofilament.

\section{Groups and Drug Administration}

300 rats were divided to 7 groups: (1) sham $(n=60)$, rats had $\mathrm{I} / \mathrm{R}$ procedures, without the insertion of nanofilament to CCA; (2) I/R ( $=60)$, the rats had cerebral ischemia by ligation for $2 \mathrm{hrs}$, and reperfusion for 1 day; (3) $25 \mathrm{mg} / \mathrm{kg}$ deltonin $(\mathrm{n}=30)$, (4) $50 \mathrm{mg} / \mathrm{kg}$ deltonin $(\mathrm{n}=30)$, and (5) $100 \mathrm{mg} / \mathrm{kg}$ deltonin $(\mathrm{n}=$ $60)$. Rats were injected intraperitoneally with 25,50 , $100 \mathrm{mg} / \mathrm{kg}$ deltonin at $2 \mathrm{hrs}$ after ischemia; (6) 100 $\mathrm{mg} / \mathrm{kg}$ deltonin $+10 \mu \mathrm{M} \operatorname{LY} 294002(\mathrm{n}=30)$, the rats were slowly injected intraventricularly with $10 \mu \mathrm{L}$ $10 \mu \mathrm{M} \mathrm{LY} 294002$ at 20 mins prior to ischemia; (7) $100 \mathrm{mg} / \mathrm{kg}$ deltonin $+5 \mu \mathrm{M}$ anisomycin $(\mathrm{n}=30)$, the rats were slowly injected intraventricularly with $10 \mu \mathrm{L} 5$ $\mu \mathrm{M}$ anisomycin at 20 mins prior to ischemia. Deltonin was dissolved in ethanol and saline to reach an ethanol concentration of 5\%. ${ }^{12}$ LY294002 and anisomycin were dissolved in $5 \%$ ethanol. Rat in sham and $\mathrm{I} / \mathrm{R}$ groups had same volume of $5 \%$ ethanol in normal saline. After $24 \mathrm{hrs}$ reperfusion, rats were anesthetized and decapitated. Figure 1B shows the experiment protocols.

\section{Assessments of Infarct Volumes, Neurological Deficits and Brain Water Contents}

After reperfusion, the rats $(n=6)$ were anesthetized and decapitated. The brains were dissected and sliced (5 slices of $1.5-\mathrm{mm}$ tissues). They were stained with $1 \%$ TTC for $30 \mathrm{~min}$ and fixed by $4 \%$ paraformaldehyde. We recorded the stained parts and calculated the volumes by ImageJ. Neurological deficits were evaluated: 0 , rat had normal behavior; 1 , rat cannot fully stretch left front leg; 2, rat turns around to a circle; 3 , rat falls to the left; 4 , rat cannot move, and lost consciousness. Brain water contents $(n=6)$ were detected at $24 \mathrm{hrs}$ after reperfusion. We measured infarct brain hemispheres as wet weight, dried them overnight at 105 to get dry weight. Brain water was calculated as [(wet weight - dry weight) $/$ wet weight $] \times 100 \%$.

\section{Rotarod Test}

Rotarod test system (TSE, Germany) evaluated neurological functions. ${ }^{13}$ Rat was trained for 3 days prior to surgeries. The speed was accelerated from 4 to $40 \mathrm{rpm}$ in 5 mins. Prior to or after $I / R / R$, each rat was detected for triplicates. We recorded the time needed for them to run on the rods.

\section{ELISA}

ELISA was utilized to measure the protein expressions of IL-6, IL-10, TNF- $\alpha$, and iNOS. Firstly, we extracted the brain tissues from ischemic area, homogenized them $(10 \%, w / v)$, and had centrifugation at 2,500 $\mathrm{r} / \mathrm{min}$ for $10 \mathrm{mins}$. In the supernatants, the protein concentration was detected by ELISA. We visualized the results through the Multiskan MK3 (Thermo Scientific, USA).

\section{Western Blotting}

We isolated the hippocampus wand measured the protein concentration via BCA detection kit (Beyotime, China). The proteins were separated via 10 SDS-PAGE and electro-transferred to a nitrocellulose membrane. The membrane was blocked by $5 \%$ skim milk. After washing, it was treated with anti-Akt (1:500), anti-p-Akt (1:500), anti-mTOR (1:500), anti-mTOR (1:500), antiP38 (1:600), anti-p-P38 (1:600), anti-LC3 (1:1000), antiBeclin-1 (1:1000), anti-MAP-2 (1:1000), anti-TLR4 (1:1000) and anti-IL-1 (1:1000). All were purchased from Abcam, USA. Then, it was incubated with secondary antibody for $2 \mathrm{hrs}$. We visualized the signals via Odyssey system (LI-COR, USA) and analyzed by ImageJ.

\section{Statistical Analysis}

We showed the data as the means \pm standard deviation (S.D.). The comparisons were carried out by one-way ANOVA with Holm-Sidak post hoc test. Differences were regarded to be significant as $\mathrm{P}<0.05$.

\section{Results}

\section{Deltonin Improved Neurological Function and Reduced Cerebral Infarction}

Compared with the sham, a great deficit was detected in $\mathrm{I} / \mathrm{R}(\mathrm{P}<0.01)$, which were markedly relieved by deltonin $(\mathrm{P}<0.05, \mathrm{P}<0.01)$ (Figure $2 \mathrm{~A}$ and $\mathrm{B}$ ). From Figure $2 \mathrm{C}$ and $\mathrm{D}$, we observed that cerebral $\mathrm{I} / \mathrm{R}$ greatly 
A

C
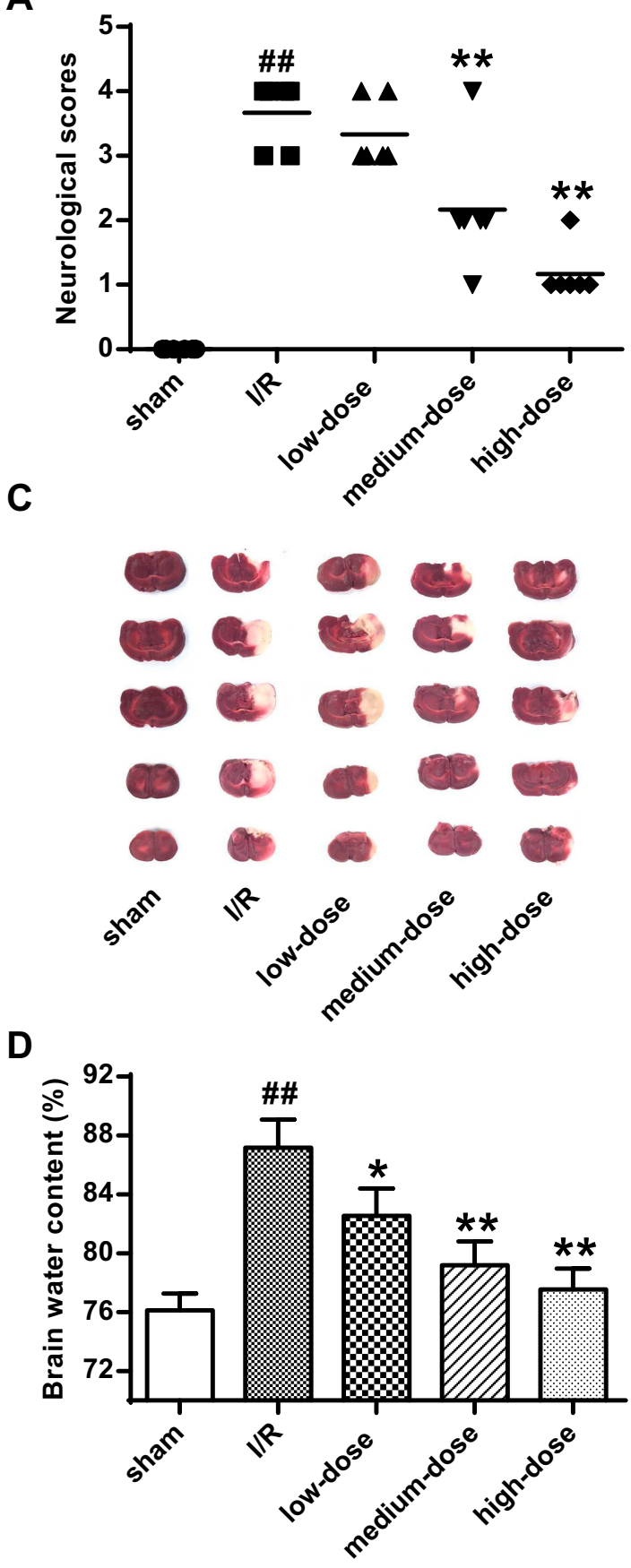

B

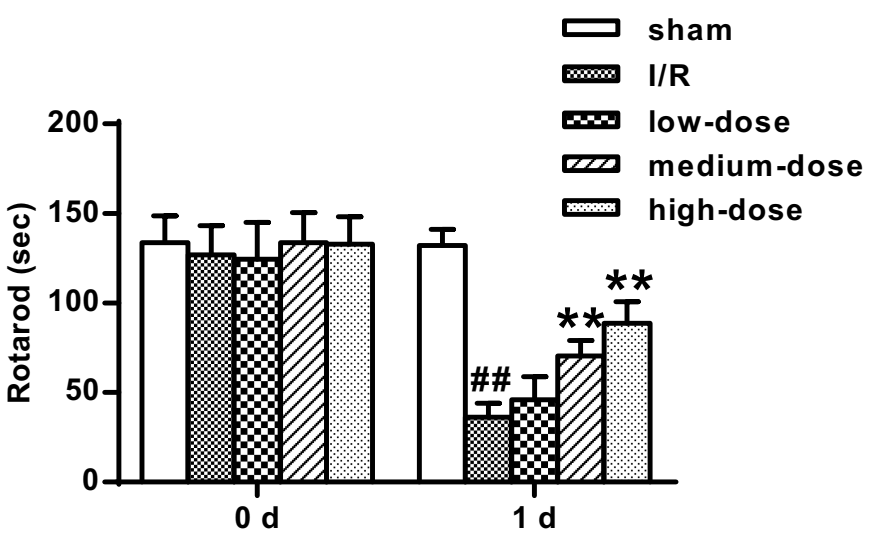

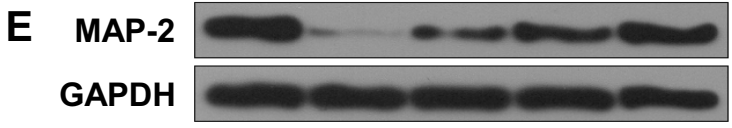

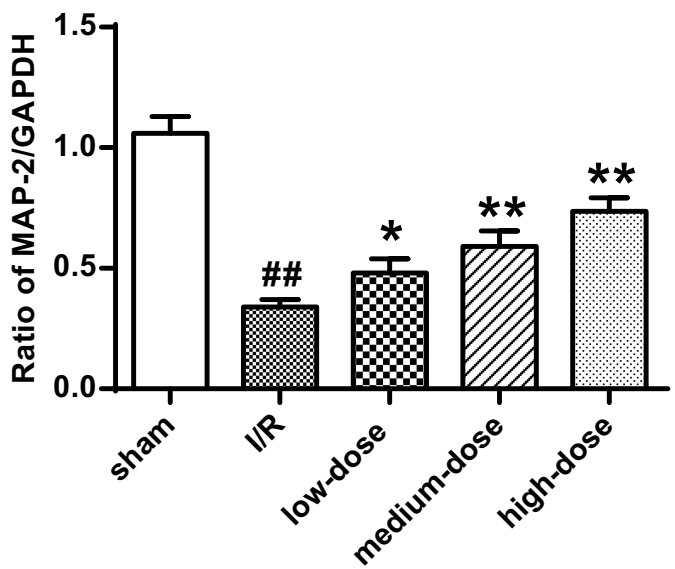

Figure 2 Deltonin improved neurological function and reduced cerebral infarction. For groups of sham, l/R, low-dose deltonin ( $25 \mathrm{mg} / \mathrm{kg})$, medium-dose deltonin ( $50 \mathrm{mg} /$ $\mathrm{kg}$ ), and high-dose deltonin (100 mg/kg): (A) Neurological scores. (B) Rotarod periods prior to or after treatments. (C) Pictures on the infarct volumes and infarct volume percentage. (D) Brain water contents. (E) Western blot of ratios of MAP-2/GAPDH. ${ }^{\# P} \mathrm{P}<0.0 \mathrm{I}$ vs sham; ${ }^{*} \mathrm{P}<0.05, * * \mathrm{P}<0.0 \mathrm{I}$ vs I/R.

elevated infarct volumes and brain water contents $(\mathrm{P}<0.01)$; however, this effect was rescued by deltonin $(\mathrm{P}<0.05, \mathrm{P}<0.01)$. Figure $2 \mathrm{E}$ shows the Western blot results. We found that MAP-2 in $\mathrm{I} / \mathrm{R}$ was much lower compared with sham $(\mathrm{P}<0.01)$. Deltonin elevated concentrations of MAP-2 $(\mathrm{P}<0.05, \mathrm{P}<0.01)$. It was obvious that deltonin improved neurological function and reduced cerebral infarction in the rats after I/R. 


\section{Deltonin Reduced Autophagy Activities Through PI3K/Akt/mTOR Signaling}

In Figure 3A, it was demonstrated that LY294002 (inhibitor of PI3K/Akt) was markedly decreased in the neurological score, when comparing with deltonin group $(\mathrm{P}<0.05)$. Figure $3 \mathrm{~B}$ shows the infarct volumes from the groups of sham, I/R, high-dose deltonin, and high-dose deltonin + LY294002. It was obvious that deltonin could reduce the infarct volumes. Figure 3C and D showed the Western blot. No obvious difference was observed in expressions of Akt and mTOR $(P>0.05)$. Compared with sham, $\mathrm{I} / \mathrm{R}$ decreased the expressions of p-Akt and p-mTOR, and increased the levels of LC3II and Beclin-1. Due to this reason, ratios of LC3-II/LC3-I and Beclin-1/GAPDH were increased ( $<$ 0.01) (Figure 3D). However, deltonin had a great elevation in $\mathrm{p}$-Akt and p-mTOR in contrast with $\mathrm{I} / \mathrm{R}(\mathrm{P}<0.01)$ (Figure 3D). In addition, the LC3-II/LC3-I and Beclin-1/ GAPDH ratios reduced greatly by deltonin $(\mathrm{P}<0.01)$ (Figure 3D). However, LY294002 greatly decreased p-Akt and pmTOR expressions and elevated the ratios of LC3-II/ LC3-I and Beclin-1/GAPDH than the $\mathrm{I} / \mathrm{R}+$ deltonin $(\mathrm{P}<0.05)$ (Figure 3D). The data indicated that deltonin attenuated autophagies and had neuroprotection to rats with $\mathrm{I} / \mathrm{R}$.

\section{Deltonin Suppressed Inflammations Through TLR4/P38/MAPK in I/R Rats}

From Figure 4, the ultrastructural changes among sham, I/R, high-dose deltonin, and high-dose deltonin+LY294002 showed that deltonin reduced autophagy activities during cerebral I/R injury. Figure 5A demonstrated that anisomycin (activator of $\mathrm{p} 38$ /MAPK) decreased the neurological scores than deltonin group $(\mathrm{P}<0.05)$. Figure 5B showed that deltonin reduced the infarct volume, but anisomycin attenuated the effect. Figure $5 \mathrm{C}$ showed the protein expressions of TLR4, p-38, p-p38, and IL-1. No significant difference was found in the expressions of $\mathrm{p} 38$ in all groups $(\mathrm{P}>0.05)$, but TLR4, p-p38, and IL-1 were elevated greatly in I/R $(P<0.01)$. Reversely, TLR4, p-p38, and IL-1 reduced by deltonin
A

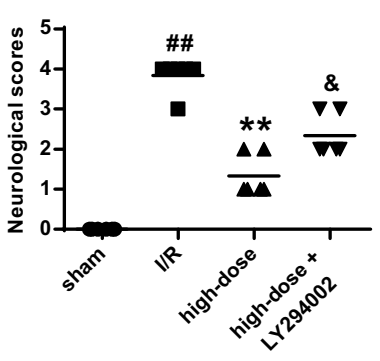

D
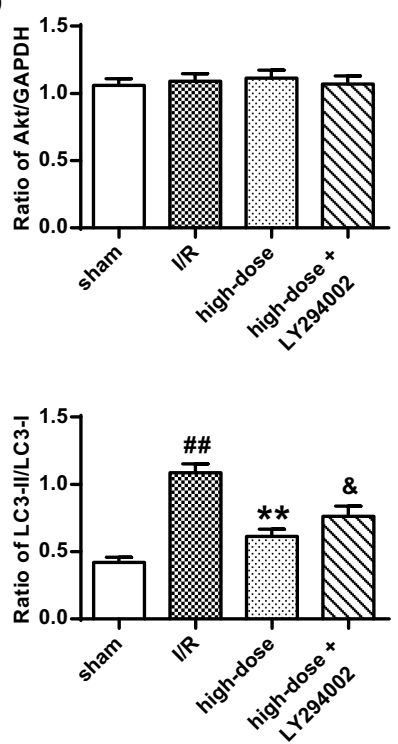

B
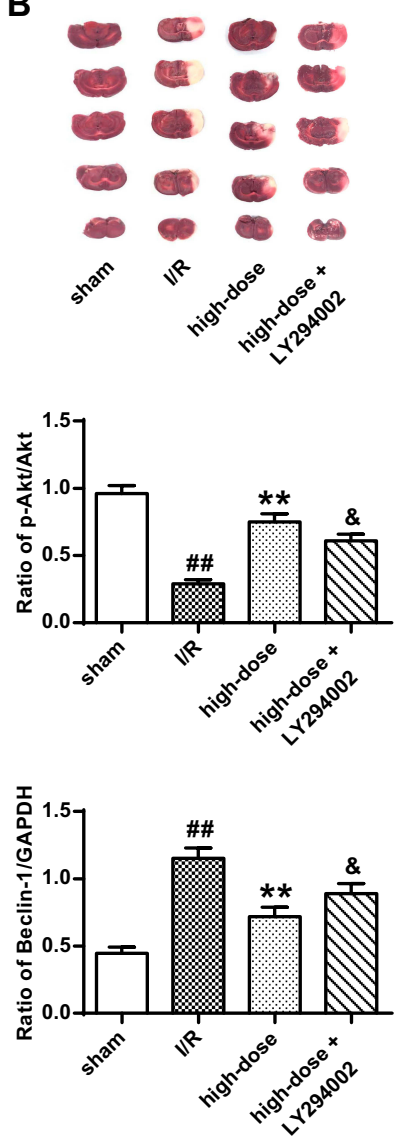
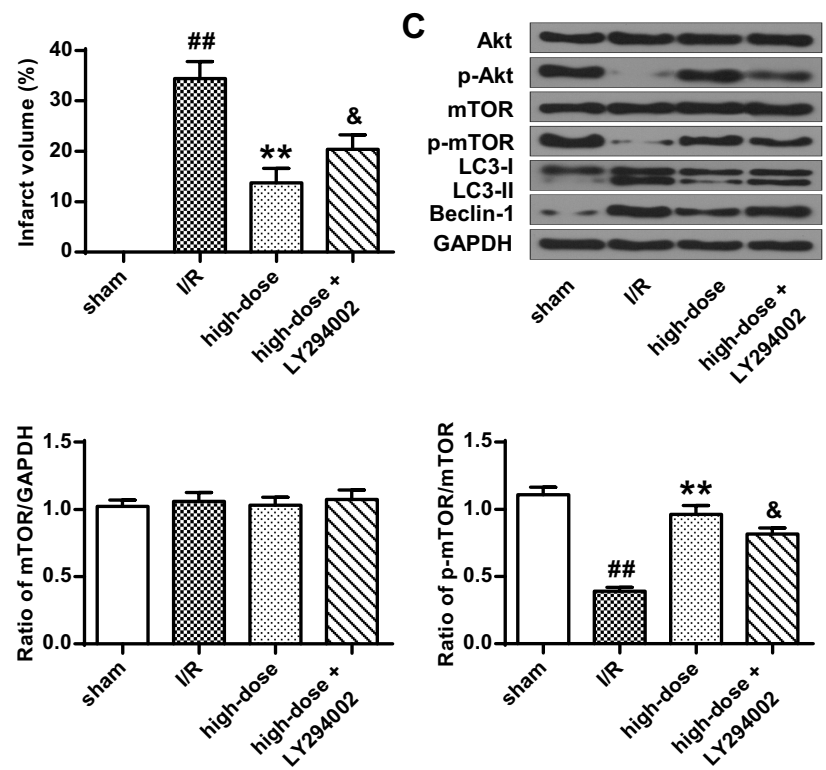

Figure 3 Deltonin reduced autophagy activities through PI3K/Akt/mTOR signaling. For groups of sham, I/R, high-dose deltonin (I00 mg/kg) and high-dose+ LY294002: (A) Neurological scores. (B) Infarct volumes. (C) and (D) Western blot images for protein expressions and ratios of Akt/GAPDH, p-Akt/GAPDH, mTOR/GAPDH, p-mTOR/ GAPDH, LC3-II/GAPDH, LC3-I/GAPDH and Beclin-I/GAPDH. ${ }^{\#} \mathrm{P}<0.01$ vs sham; ${ }^{*}$ P $<0.01$ vs I/R; ${ }^{\&} \mathrm{P}<0.05$ vs deltonin. 


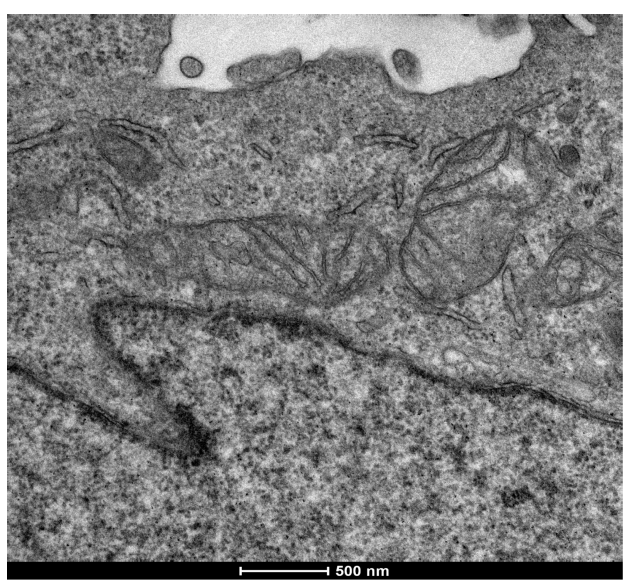

sham

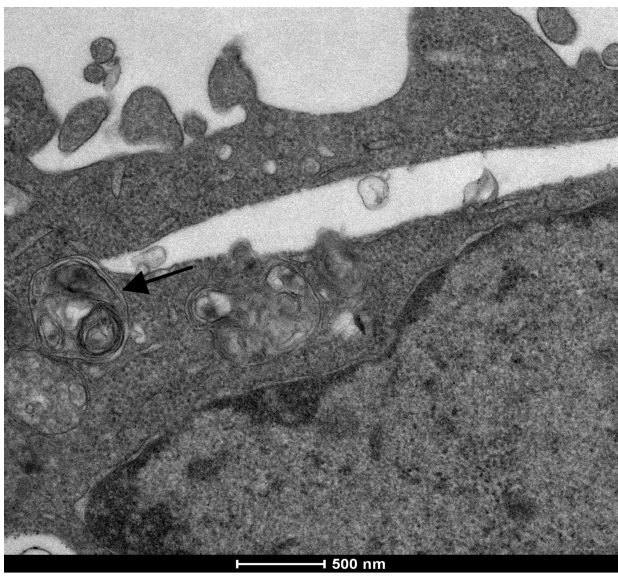

high-dose

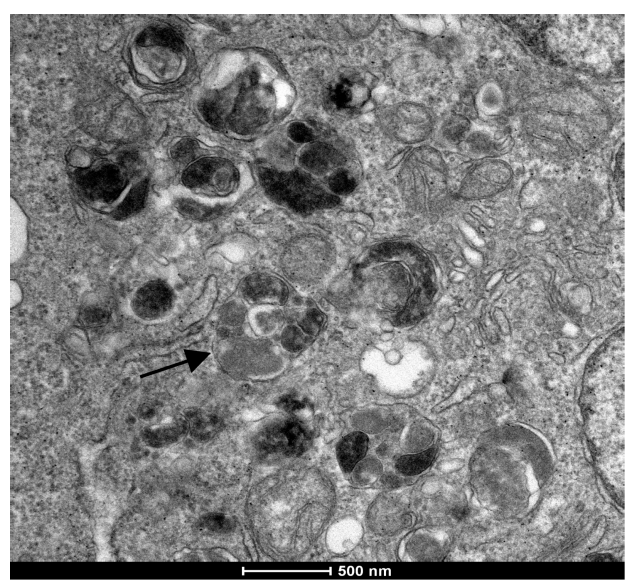

I/R

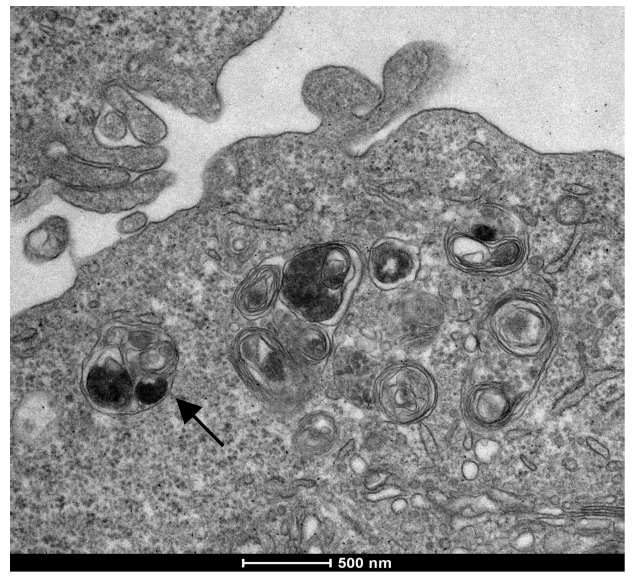

high-dose + LY294002

Figure 4 TEM images showing the ultrastructural changes. The ultrastructural changes among sham, I/R, high-dose deltonin, and high-dose deltonin+LY294002 showed that deltonin reduced autophagy activities during cerebral I/R injury.

$(\mathrm{P}<0.01)$ (Figure 5C and D). However, anisomycin up-regulated the expressions of TLR4, p-p38, and IL-1 $(\mathrm{P}<0.05)$.

Figure 6 showed the ELISA results about inflammatory factors of IL-6 (Figure 6A), IL-10 (Figure 6B), TNF- $\alpha$ (Figure 6C), and iNOS (downstream to TLR4) (Figure 6D). It was noted that IL- $6, \mathrm{TNF}-\alpha$, and iNOS were elevated greatly after $\mathrm{I} / \mathrm{R}(\mathrm{P}<0.01)$. But IL-10 was markedly inhibited than sham $(\mathrm{P}<0.01)$. With $100 \mathrm{mg} / \mathrm{kg}$ deltonin, IL-6, TNF- $\alpha$, and iNOS were greatly decreased, but IL-10 was markedly promoted than $\mathrm{I} / \mathrm{R}$ rats $(\mathrm{P}<0.01)$. But LY294002 and anisomycin up-regulated the IL-6, TNF- $\alpha$, and iNOS and downregulated IL-10 $(\mathrm{P}<0.01)$. It was identified that deltonin attenuated the inflammation impacts of $I / R$ in rats.

\section{Discussions}

It was well established that neurological functions, infarct volumes and brain water contents are the major indicators for cerebral ischemia/reperfusion injuries. An effective treatment could usually pose an impact in the three factors. For example, Y. Lin reported the neuroprotection effects from resveratrol on I/R injuries in rat by TRPC6/CREB. ${ }^{14}$ Resveratrol lowered the neurological disorders and decreased brain infarctions at 1 day after reperfusions Microtubule-Associated Protein-2 (MAP-2) is a neuronspecific phosphoprotein which is measured as a marker of ischemic injury following cerebral ischemia. ${ }^{15}$ In this study, we found that MAP-2 in I/R was much lower compared with sham, and deltonin elevated concentrations of MAP-2. Our study results are in agreement with the results of the studies by previous researches which represented that MAP-2 decreased during cerebral I/R. ${ }^{14,16}$ In our experiments, we noticed a great deficit was detected in $\mathrm{I} / \mathrm{R}$, which were markedly relieved by deltonin, compared with the sham. We observed that cerebral $I / R$ greatly 


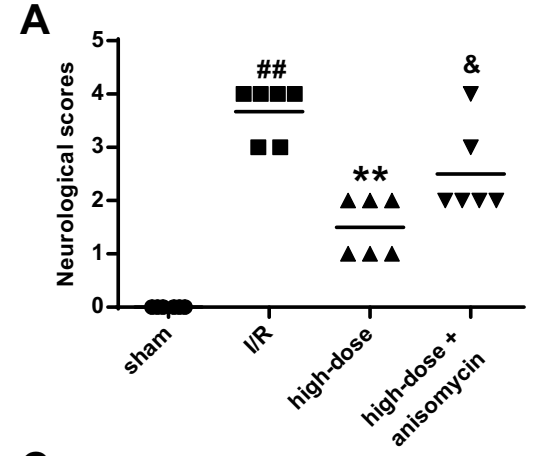

C
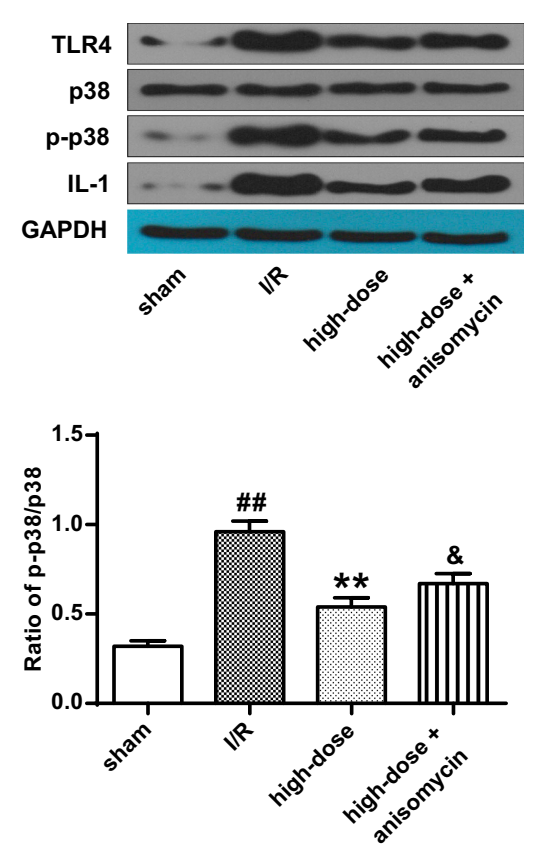

B

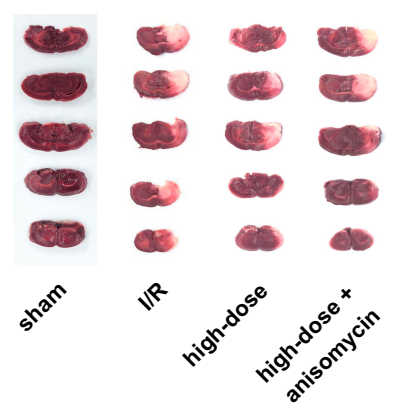

D
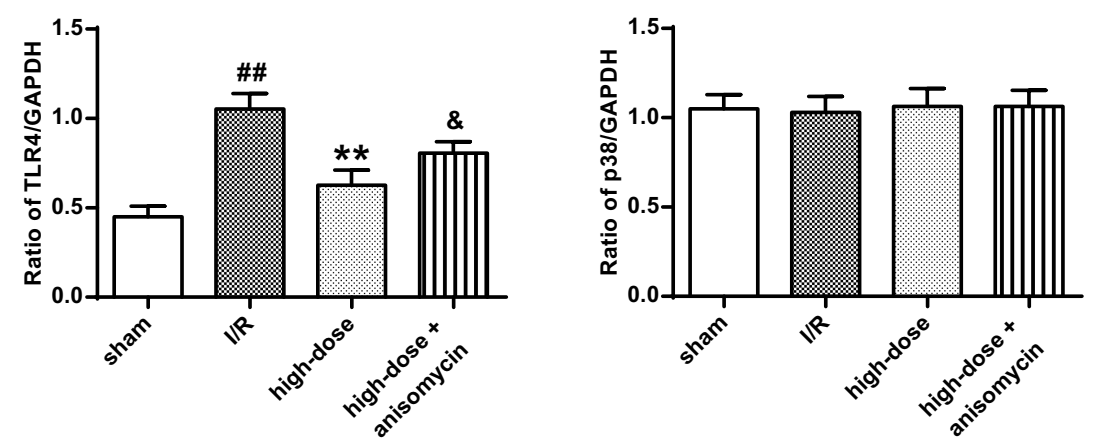

Figure 5 Deltonin suppressed inflammations through TLR4/p38/MAPK in I/R rats. For groups of sham, I/R, high-dose deltonin (I00 mg/kg) and high-dose+ anisomycin: (A) Neurological scores. (B) Infarct volumes. (C) Western blot images for protein expressions of TLR4, p38, p-P38, IL-I, and GAPDH. (D) Ratios of TLR4/GAPDH, P38/ GAPDH, P-P38/GAPDH, and IL-I/GAPDH. ${ }^{\#} \mathrm{P}<0.01$ vs sham; ${ }^{* *} \mathrm{P}<0.01$ vs I/R; ${ }^{8} \mathrm{P}<0.05$ vs deltonin.

elevated infarct volumes and brain water contents; however, this effect was rescued by deltonin. It was obvious that deltonin improved neurological function and reduced cerebral infarction in the rats after $\mathrm{I} / \mathrm{R}$.

According to Sai Ma in 2015, autophagy activation during cardiac $\mathrm{I} / \mathrm{R}$ could either antagonize cardiac pathogenesis or contribute to further myocardium damage. ${ }^{17}$ In our experiments, I/R increased the levels of LC3II and Beclin-1, which were 2 autophagy indicators. I/R also decreased the expressions of $\mathrm{p}$-Akt and p-mTOR. However, deltonin had a great elevation in p-Akt and p-mTOR in contrast with $\mathrm{I} / \mathrm{R}$, and in addition, reduced the expressions of LC3-II and Beclin-1 greatly. Moreover, LY294002, an indicator of PI3K/Akt, greatly elevated the ratios of LC3-II Beclin-1. The data indicated that deltonin reduced autophagy and had neuroprotection to rats with $\mathrm{I} / \mathrm{R}$ through $\mathrm{PI} 3 \mathrm{~K} / \mathrm{Akt} /$ mTOR signaling.

TLR4/p38/MAPK signaling pathway was widely known to participate in the activation of inflammations. For instance, J. Li demonstrated that oridonin protected against the inflammatory response in diabetic nephropathy by inhibiting the TLR4/p38-MAPK and TLR4/NF- $\kappa B$ signaling pathways. ${ }^{18}$ In 2018, JP. Dai also reported that oxymatrine inhibited influenza a virus replication and inflammation via TLR4, p38 MAPK and NF-кB Pathways. ${ }^{19}$ There were no significant differences found in the expressions of p38 in all groups, but TLR4, p-p38, and IL-1 were elevated greatly in I/R group. Reversely, TLR4, p-p38, and IL-1 reduced by deltonin. But anisomycin, that could activate p38/MAPK, up-regulated the expressions of TLR4, p-p38, and IL-1. 
A

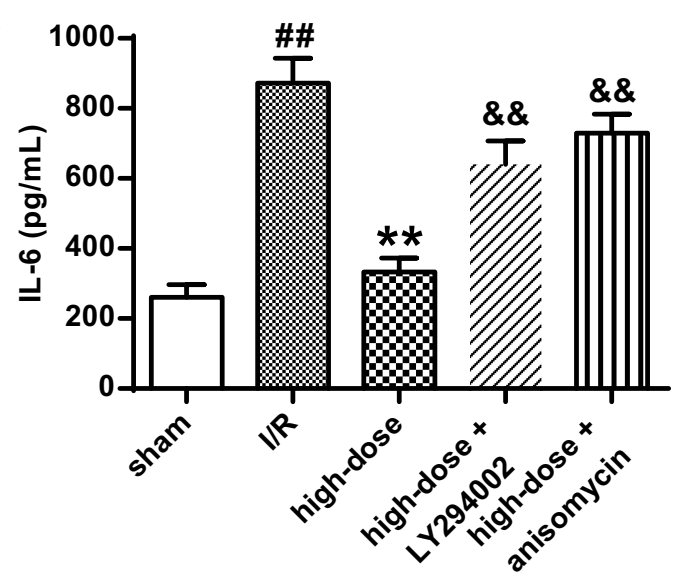

C

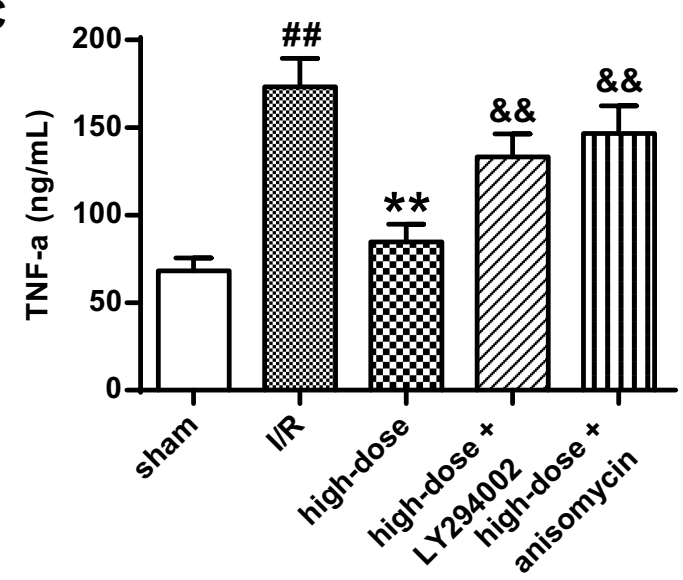

B

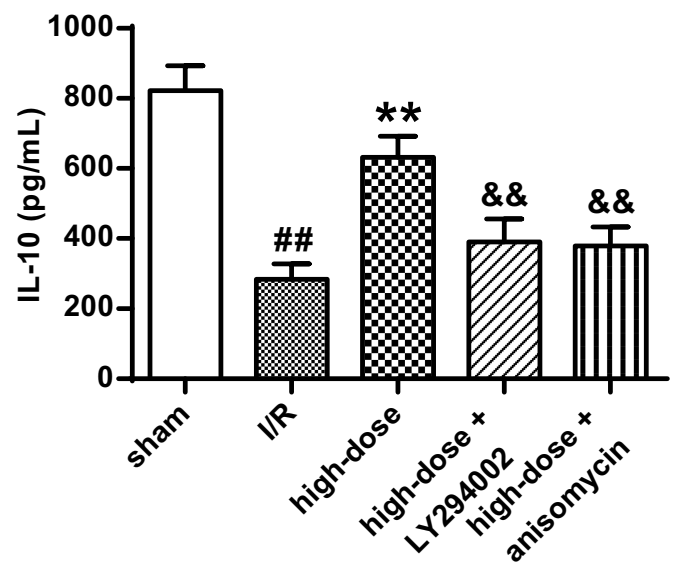

D

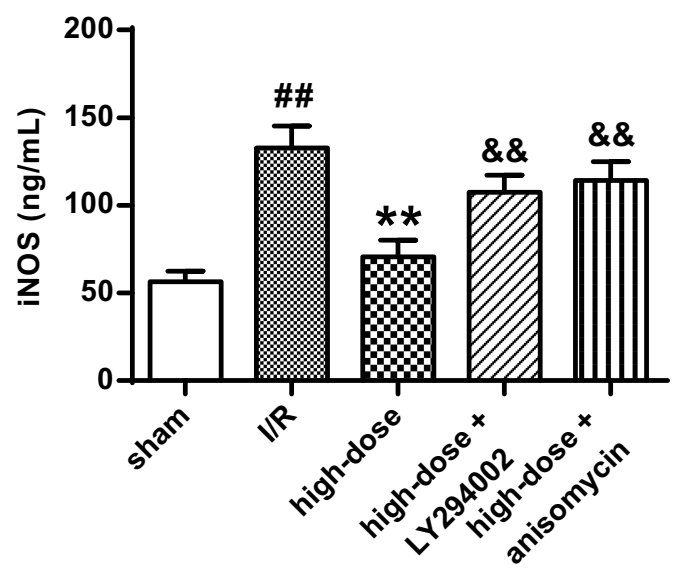

Figure 6 Protein expressions of IL-6 (A), IL-10 (B), TNF- $\alpha$ (C), and iNOS (D) for groups of sham, I/R, high-dose deltonin (I00 mg/kg), high-dose+ LY294002, and high-dose + anisomycin. ${ }^{\#} \mathrm{P}<0.01$ versus sham group; ${ }^{* *} \mathrm{P}<0.0$ I versus I/R group; ${ }^{\&} \mathrm{P}<0.0$ I versus deltonin group.

In addition, we detected the protein expressions of inflammatory factors were elevated greatly by $\mathrm{I} / \mathrm{R}$, and IL-10 was markedly reduced. By $100 \mathrm{mg} / \mathrm{kg}$ deltonin, IL-6, TNF- $\alpha$, and iNOS were greatly decreased, but IL-10 was markedly promoted than I/R rats. But LY294002 and anisomycin up-regulated the IL- 6 , TNF- $\alpha$, and iNOS and down-regulated IL-10 in the $\mathrm{I} / \mathrm{R}+\mathrm{LY} 294002+$ deltonin and $\mathrm{I} / \mathrm{R}+$ anisomycin+deltonin groups than the $\mathrm{I} / \mathrm{R}+$ deltonin group. Obviously, deltonin attenuated the inflammation impacts of I/R through TLR4/p38/ MAPK signaling pathway.

\section{Conclusions}

Deltonin could suppress ischemic brain injury by regulating autophagy and inflammation during I/R. Deltonin can be an efficient therapeutic method for patient with ischemia.

\section{Data Sharing Statement}

The analyzed data sets generated during the study are available from the corresponding author on reasonable request.

\section{Ethics Approval}

The present study was approved by the Ethics Committee of Fu-Ning People's Hospital.

\section{Author Contributions}

All authors contributed to data analysis, drafting or revising the article, gave final approval of the version to be published, and agree to be accountable for all aspects of the work.

\section{Disclosure}

The authors declare that they have no conflicts of interest in this work.

\section{References}

1. Tong Q-Y, Qing Y, Shu D, et al. Deltonin, a steroidal saponin, inhibits colon cancer cell growth in vitro and tumor growth in vivo via induction of apoptosis and antiangiogenesis. Cell Physiol Biochem. 2011;27 (3-4):233-242. doi:10.1159/000327949 
2. Zhang S, He Y, Tong Q, Chen Q, Wu X, Huang W. Deltonin induces apoptosis in MDA-MB-231 human breast cancer cells via reactive oxygen species-mediated mitochondrial dysfunction and ERK/AKT signaling pathways. Mol Med Rep. 2013;7(3):1038-1044. doi:10.3892/mmr.2013.1273

3. Xie Y, Fan M, Jiang R, Wang Z, Li Y. Deltonin induced both apoptosis and autophagy in head and neck squamous carcinoma $\mathrm{FaDu}$ cell. Neoplasma. 2015;62(3):419-431. doi:10.4149/neo_2015_050

4. Zhang X, Yan H, Yuan Y, et al. Cerebral ischemia-reperfusioninduced autophagy protects against neuronal injury by mitochondrial clearance. Autophagy. 2013;9(9):1321-1333. doi:10.4161/auto.25132

5. Xin X-Y, Pan J, Wang X-Q, et al. 2-Methoxyestradiol attenuates autophagy activation after global ischemia. Can J Neurol Sci. 2011;38(4):631-638. doi:10.1017/S031716710001218X

6. Rami A, Langhagen A, Steiger S. Focal cerebral ischemia induces upregulation of Beclin 1 and autophagy-like cell death. Neurobiol Dis. 2008;29(1):132-141. doi:10.1016/j.nbd.2007.08.005

7. Carloni S, Buonocore G, Balduini W. Protective role of autophagy in neonatal hypoxia-ischemia induced brain injury. Neurobiol Dis. 2008;32(3):329-339. doi:10.1016/j.nbd.2008.07.022

8. Gao L, Jiang T, Guo J, et al. Inhibition of autophagy contributes to ischemic postconditioning-induced neuroprotection against focal cerebral ischemia in rats. PLoS One. 2012;7(9):e46092. doi:10.1371/ journal.pone.0046092

9. Zhang J, Wang C, Yu S, et al. Sevoflurane postconditioning protects rat hearts against ischemia-reperfusion injury via the activation of PI3K AKT/mTOR signaling. Sci Rep. 2014;4:7317. doi:10.1038/srep07317

10. Chen A, Xiong L-J, Tong Y, Mao M. Neuroprotective effect of brainderived neurotrophic factor mediated by autophagy through the PI3K/ Akt/mTOR pathway. Mol Med Rep. 2013;8(4):1011-1016. doi: $10.3892 / \mathrm{mmr} .2013 .1628$

11. Yasuda N, Ishii T, Oyama D, et al. Neuroprotective effect of nobiletin on cerebral ischemia-reperfusion injury in transient middle cerebral artery-occluded rats. Brain Res. 2014;1559:46-54. doi:10.1016/j. brainres.2014.02.007
12. Li M, Qu YZ, Zhao ZW, et al. Astragaloside IV protects against focal cerebral ischemia/reperfusion injury correlating to suppression of neutrophils adhesion-related molecules. Neurochem Int. 2012;60 (5):458-465. doi:10.1016/j.neuint.2012.01.026

13. Zausinger S, Hungerhuber E, Baethmann A, Reulen H-J, SchmidElsaesser R. Neurological impairment in rats after transient middle cerebral artery occlusion: a comparative study under various treatment paradigms. Brain Res. 2000;863(1-2):94-105. doi:10.1016/ S0006-8993(00)02100-4

14. Lin Y, Chen F, Zhang J, et al. Neuroprotective effect of resveratrol on ischemia/reperfusion injury in rats through TRPC6/CREB pathways. J Mol Neurosci. 2013;50(3):504-513. doi:10.1007/s12031013-9977-8

15. Dawson DA, Hallenbeck JM. Acute focal ischemia-induced alterations in MAP2 immunostaining: description of temporal changes and utilization as a marker for volumetric assessment of acute brain injury. J Cereb Blood Flow Metab. 1996;16(1):170-174. doi:10.109 7/00004647-199601000-00020

16. Lin Q, Hai J, Yao L-Y LY. Neuroprotective effects of NSTyr on cognitive function and neuronal plasticity in rats of chronic cerebral hypoperfusion. Brain Res. 2010;1325:183-190. doi:10.1016/j.brainres. 2010.02.037

17. Ma S, Wang Y, Chen Y, Cao F. The role of the autophagy in myocardial ischemia/reperfusion injury. Biochim Biophys Acta Mol Basis Dis. 2015;1852(2):271-276. doi:10.1016/j.bbadis.2014. 05.010

18. Li J, Bao L, Zha D, et al. Oridonin protects against the inflammatory response in diabetic nephropathy by inhibiting the TLR4/p38-MAPK and TLR4/NF- $\mathrm{KB}$ signaling pathways. Int Immunopharmacol. 2018;55:9-19. doi:10.1016/j.intimp.2017.11.040

19. Dai J-P, Wang Q-W, Su Y, et al. Oxymatrine inhibits influenza A

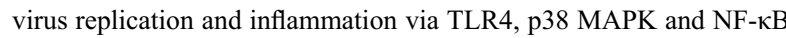
pathways. Int J Mol Sci. 2018;19(4):965. doi:10.3390/ijms19040965
Neuropsychiatric Disease and Treatment

\section{Publish your work in this journal}

Neuropsychiatric Disease and Treatment is an international, peerreviewed journal of clinical therapeutics and pharmacology focusing on concise rapid reporting of clinical or pre-clinical studies on a range of neuropsychiatric and neurological disorders. This journal is indexed on PubMed Central, the 'PsycINFO' database and CAS, and is the official journal of The International Neuropsychiatric Association (INA). The manuscript management system is completely online and includes a very quick and fair peer-review system, which is all easy to use. Visit http://www.dovepress.com/testimonials.php to read real quotes from published authors. 